\title{
Prescription for Progress- Enhancing Communication between Pharmacists and Patients
}

\author{
Kenric B Ware* \\ Department of Pharmacy Practice, South University School of Pharmacy, South University, Science Court, Columbia, USA
}

Received: December 5, 2013; Accepted: January 28, 2014; Published: January 29, 2014

*Corresponding author: Kenric B Ware, Department of Pharmacy Practice, South University School of Pharmacy, South University, Science Court, Columbia, SC 29203, USA, Tel: 803-935-9695; Fax: 803-935- 4564; E-mail: kbware@southuniversity.edu

\begin{abstract}
The provision of sound instructions to patients with regards to adherence to their respective medication therapies is a key responsibility of pharmacists. While this functionality is irrefutably important, actualizing these processes is difficult. The multifaceted demands of pharmacists in various practice settings, coupled with fluctuating literacy levels displayed by patients, render productive communication between pharmacists and patients consistently under construction. As a result, common obstacles to effective dialogue will be reviewed here. The impact that these occurrences have on maximizing therapeutic benefits for patients will also be discussed.
\end{abstract}

\section{Prescription for progress}

The ability for patients to achieve adherence with their medication therapies is often a daunting task. Patients rely on the expertise of pharmacists to help them navigate this process. Appropriate guidance hinges upon the ability to identify patients' needs and work to fill gaps in knowledge. The purpose of this address is to provide practical steps for improving communication with patients.

Possessing the ability to express oneself and truly understand another's point of view is complex. It extends beyond the parameters of passively listening with frequent retorts of "yes" or "no". Aspirations to effectively communicate mandates full engagement in the process. Communication is accomplished verbally and nonverbally as expressions and dispositions have a tendency to form their own sentences. Pharmacists are not immune to the difficulties that communication presents. These challenges for pharmacists often manifests through interactions with patients regarding their respective medication therapies.

Pharmacists are cautioned to ensure that their behaviors and mannerisms convey messages they are intending to transmit. Patients are often regarded as vulnerable considering they are generally heavily reliant upon the information being disseminated. With many patients lacking the wherewithal to serve as active participants in their own care, pharmacists assume incredible responsibility for competent and compassionate delivery. This delivery is frequently riddled by speaking in terms that are incomprehensible to recipients.
A more focused effort has to be made to differentiate conversation appropriate for other health care professionals as opposed to dialogue more suitable for patients. Efforts to standardize tools for better communication have been undertaken with reasonable success [1]. However, barriers between pharmacists and patients persists that warrant further exploration. These include but are not limited to lack of clarification in providing instructions, sarcasm and jargon, use of acronyms, and ineffective listening skills.

\section{Invite questions from patients}

The reliable and relevant adage remarks "....With all thy getting, get understanding." Understanding in its purest form is often difficult to apprehend.

Patients in essence transition to a place of innate fear of ridicule when asking for guidance. Perhaps their uneasiness stems from the perception that interrogatives denote inattentiveness. A potential mechanism that pharmacists can employ to combat these occurrences is to create an environment conducive to conversing. This may be accomplished by insisting that patients pose questions about things that they find to be unclear.

\section{Avoid sarcasm}

Sarcasm is often an understated barrier to communication. When pharmacists assume this type of dialogue with their patients they are frequently misunderstood. It is very uncertain whether or not the speaker is expressing his or her true concern or making light of the situation. Sarcasm can be difficult to curtail if the practice has grown to become habitual. Pharmacists have to continue being vigilant to guard against such discourses.

\section{Minimize acronym usage}

There has been and continues to be an explosion in the incorporation of acronyms into daily conversations. An untoward effect of our "acronym flurry" society is that the same acronym can be used to refer to multiple descriptions. A patient overhearing practitioners referring to their condition as SOB (Shortness of Breath) may instinctively regard the comment as derogatory in nature. It is understandable how patients can become easily intimidated by the cryptic language. A practical 
remedy may reside in generating a list of approved acronyms for patients on behalf of authorized personnel.

\section{Improvement of listening skills}

Pharmacists that are hampered by ineffective listening also play a large role in contaminated communications. This undesirable trait may arise from mentally preparing to talk as opposed to absorbing information presented. Patients may incorrectly deduce that their concerns are insignificant and should be ignored. In addition to pharmacists not being privy to clues that may assist in modifying drug therapies, the trust and allegiance that patients strive to develop is punctured. Avidly working to minimize distractions that are prone to interrupt pharmacists during their exchanges with patients is vital.

\section{Conclusion}

Pharmacists' command of communicating with patients is a purposeful work in progress. Highlighting strengths in communication skills without addressing limitations provides a false sense of improvement. Effective communication is essential for adequate patient care; if properly conditioned, pharmacists may turn it into a delightful exercise.

\section{Reference}

1. Johnston J, Fidelie L, Robinson K, Killion J, Behrens P (2012) An instrument for assessing communication skills of healthcare and human services students. Internet Journal of Allied Health Sciences and Practice 10: 1-6. 